\title{
El feto como paciente: diferentes posturas sobre un mismo concepto
}

\section{The fetus as a patient: different positions on the same concept}

\author{
Milagros Ma. de las Mercedes Moreno D’Anna, * Gustavo Páez **
}

https://doi.org/10.36105/mye.2021v32n4.03

\section{Resumen}

Las malformaciones anatómicas congénitas, como los trastornos genéticos, son causa actual y frecuente de aborto de tipo eugenésico en países con despenalización y/o legalización del aborto. La medicina fetal diagnóstica y terapéutica, entre las que se encuentra la cirugía fetal intrauterina, ha colocado en el universo de la ciencia biomédica al feto como un nuevo paciente. Plantear que el feto es un paciente significaría reconocer que es una persona. Para saber si un feto es un paciente o no, se debe establecer si éste tiene un estado moral independiente. En el presente artículo analizaremos tres posturas sobre la consideración del feto como paciente.

Palabras clave: feto, persona, paciente, cirugía fetal, aborto eugenésico, personalismo, principialismo.

\footnotetext{
* Universidad Austral, Facultad de Ciencias Biomédicas, Departamento de Bioética. Argentina. Correo electrónico: mdmoreno@austral.edu.ar https://orcid.org/0000-0001-8647-2591

** Universidad Austral, Facultad de Ciencias Biomédicas, Departamento de Bioética. Argentina. Correo electrónico: gpaez@austral.edu.ar https://orcid.org/0000-0003-3862-7426

Recepción: 10 de mayo de 2021. Aceptación: 20 de junio de 2021.
} 


\section{Introducción}

Las malformaciones anatómicas congénitas, como los trastornos genéticos, son causa actual y frecuente de aborto de tipo eugenésico en países con despenalización y/o legalización del aborto. En España, en 1985 ya se aprueba el aborto cuando «se presuma que el feto habrá de nacer con graves taras físicas o psíquicas, siempre que el aborto se practique dentro de las veintidós primeras semanas de gestación...» (1).

Con el advenimiento de las diferentes técnicas de diagnóstico prenatal, se ha logrado evaluar al no nacido, determinar alguna enfermedad y establecer el pronóstico (2). Este gran avance de la ciencia ha permitido dos acciones antagónicas. Una consiste en el beneficio del diagnóstico temprano de patologías que, tratadas a tiempo y de forma adecuada, pueden salvar la vida del niño por nacer y/o mejorar su calidad de vida postnatal, siendo la cirugía fetal un ejemplo de tratamiento. Pero también ha permitido que en gran medida se haya perdido la sensibilidad hacia la igual dignidad de todos los seres humanos, provocando intolerancia a que nazcan personas con una enfermedad congénita o que pudieran presentar algún tipo de discapacidad (1) o, al menos, el derecho a elegir si se desea continuar con la vida del feto (3). Como afirma Best, muchas veces la mentalidad que guía esta técnica se orienta a «aseguran» que el niño por nacer no tendrá ningún defecto congénito (4), originando una especie de presión, en caso de que el feto presente alguna discapacidad, para que sea «descartado». Incluso, en muchos casos, las madres que deciden seguir adelante con su embarazo suelen sentirse discriminadas (5).

Todo esto es consecuencia de la premisa utilitaria que considera que la pobre calidad de vida de una persona, como consecuencia de trastornos congénitos, aumenta la «cantidad total de daño» (6). Nuccetelli (2017) responde a ese presupuesto con casos concretos de pacientes con mielomeningocele, que no consideran que tengan 
mala calidad de vida. En el mismo sentido, aunque de modo más amplio, Campbell y Stramondo niegan que la incapacidad sea equivalente a mala calidad de vida (7).

La medicina fetal diagnóstica y terapéutica, entre la que se encuentra la cirugía fetal intrauterina, ha colocado al feto como un nuevo paciente en el universo de la ciencia biomédica. Es un medio para atender al feto desde los momentos iniciales de su existencia y procurar brindarle una mejor calidad de vida, sin poner en un riesgo desproporcionado a su madre.

En el presente artículo, luego de un breve recorrido histórico de los principales hitos que dieron origen a la medicina fetal y a la cirugía fetal intrauterina, nos centraremos en la consideración del feto como paciente. Como se verá más adelante, analizaremos las tres posturas que actualmente existen sobre este tema.

\section{La cirugía fetal}

La cirugía fetal es un procedimiento invasivo que se realiza en un feto dentro del útero, para ayudar a mejorar la terapia en el largo plazo para los niños con defectos congénitos específicos. Se recurre a esta técnica porque estos defectos con frecuencia empeoran a medida que se desarrolla el feto. La cirugía fetal es realizada por un equipo de expertos que se centra en el tratamiento y en la mejora de las condiciones antes del nacimiento (8).

En 1884, Cohnstein y Zuntz informaron de la primera cirugía fetal no humana, pero en la década de los 40 del siglo XX se desarrollaron técnicas que permitieron extraer del útero un feto de rata, tratarlo quirúrgicamente y regresarlo con éxito al útero, continuando el embarazo (9).

El primer informe de amniocentesis humana en la literatura fue publicado por Lambl en 1881, en Alemania, para el tratamiento del polihidramnios (10).

Medicina y Ética - Octubre-Diciembre 2021 - Vol. 32 - Núm. 4

https://doi.org/10.36105/mye.2021v32n4.03 
En 1952, Bevis utilizó la amniocentesis para determinar la gravedad de la eritroblastosis por incompatibilidad Rh (10). Fuchs y Riis utilizaron dicho procedimiento para la determinación del sexo $\mathrm{y}$ de enfermedades hereditarias.

En 1965, Liley implementa la transfusión intrauterina para subsanar la eritroblastosis por incompatibilidad Rh (9). Esta metodología se hizo más segura mediante el uso de la ecografía (10), la cual se describió por primera vez como método para evaluación obstétrica en 1968 (9). Cuando se hizo posible realizar una transfusión fetal relativamente no invasiva utilizando como guía el ultrasonido, entonces cesaron los esfuerzos por acceder al feto a través de la cirugía fetal abierta (9).

En 1972, el trabajo de Liggins y Howie demostró una reducción en más del $50 \%$ de casos de membrana hialina en prematuros nacidos vivos, al menos 24 horas después de inducir la madurez pulmonar con betametasona (11). En 1974, en la Universidad de Yale se realizó la primera visualización fetal por fetoscopía, orientándose inicialmente al diagnóstico directo, o para la obtención de biopsias (11). En 1975, Benzie y Doran utilizaron un fetoscopio para visualizar los contenidos intrauterinos antes del aborto con solución salina (10).

En 1981, la cirugía fetal pasó de ser una herramienta de diagnóstico a una herramienta terapéutica en modelos experimentales en primates. Michejda y Hodgen idearon lo que llamaron HAVIT (Hydrocephalic Antenatal Vent for Intrauterine Treatment) (10). Se probó que, con la colocación de las derivaciones ventriculares en estos primates hidrocefálicos, hubo mayor supervivencia al parto, mejores habilidades motoras y progreso ponderal postnatal. Estos modelos en primates pudieron identificar que el uso de anestésicos inhalados era un factor de riesgo que disminuía la actividad uterina (10). Del mismo modo, se reveló que las grapas metálicas disminuían en un $50 \%$ la fertilidad materna, pero también se demostró que eran posibles futuros embarazos después de la cirugía fetal $(92.6 \%)(10)$. 
La primera cirugía materno-fetal abierta reportada en humanos fue en 1982 por el Dr. Harrison (12). Se realizó una vesicostomía en un feto con hidronefrosis congénita bilateral. Desde ese momento el campo de la terapia fetal ha ganado importancia y la consideración del feto como un paciente (12).

A pesar de varios fracasos en ensayos con animales, el Dr. Michael Harrison continuó realizando investigaciones sobre cirugía fetal en corderos y monos, refinando los criterios para las diferentes intervenciones fetales (10).

En 1982 se reunieron diversos profesionales (perinatólogos, gineco obstetras, expertos en ultrasonido, pediatras, cirujanos, bioeticistas, fisiólogos) de una docena de instituciones de cinco países, para discutir el campo emergente de las terapias fetales (11). Se creó, así, la Sociedad Internacional de Medicina Fetal y Cirugía, la cual estableció los primeros criterios básicos para realizar una cirugía fetal. Éstos se enumeran a continuación:

1. Las malformaciones anatómicas adecuadas para el tratamiento in útero son defectos estructurales simples que interfieren con el desarrollo de los órganos, pero que pueden permitir que continúe un desarrollo fetal normal si se corrigen.

2. El feto debe ser único, sin anomalías estructurales ni genéticas adicionales.

3. La historia natural del defecto y la enfermedad fetal debe ser conocida, con intervención justificada, sólo si existe una probabilidad de beneficio razonable.

4. Antes de considerar una cirugía debe realizarse una cuidadosa evaluación en serie de la anatomía y función del órgano, para excluir fetos que tengan una afección lo suficientemente leve para que puedan esperar a recibir terapia postnatal, así como fetos tan severamente afectados que no puedan ser salvados.

5. La familia debe recibir asesoría acerca de los riesgos y beneficios y estar de acuerdo con el tratamiento, incluyendo seguimiento de largo plazo.

Medicina y Ética - Octubre-Diciembre 2021 - Vol. 32 - Núm. 4 
6. Un equipo multidisciplinario -que incluya un especialista en medicina materna experimentado en diagnóstico prenatal, un cirujano pediátrico y un neonatólogo-, debe acordar el plan de tratamiento.

7. Debe haber acceso a una unidad obstétrica con nivel III de alto riesgo y a una unidad neonatal de cuidados intensivos, así como a asesoría bioética y psicosocial.

En 1994, el equipo liderado por el Dr. Rubén Quintero realizó la primera ligadura del cordón umbilical a través de la fetoscopía. En 1995, el equipo del mismo investigador practicó la primera cistoscopía fetal para tratar la obstrucción vesical causada por una valva en la uretra posterior, utilizando láser (11).

Con el nuevo milenio aparecen importantes publicaciones de grupos europeos multicéntricos, como el liderado por Ian Deprest, que resaltan la potencialidad futura de esta cirugía, y hacen un recuento del avance en las principales indicaciones de ese momento, sobre todo orientada a la prevención de secuelas de las patologías no tratadas en la etapa fetal (11), o como el estudio aleatorizado y controlado MOMS del año 2011, realizado en tres centros estadounidenses, que estudia los beneficios de la cirugía fetal en pacientes con mielomeningocele.

A partir del 2010, en América Latina comienzan a formarse centros de formación y desarrollo. Algunos países, como México, Chile, Brasil, Venezuela y Argentina, han publicado importantes experiencias: en Querétaro, México, está el grupo liderado por el Dr. Rogelio Cruz; en Chile, el liderado por el Dr. Yamamoto y el Dr. Otayza y, en Argentina, el Dr. Echegaray (11).

Estudios recientes han concluido que actualmente la cirugía de mielomeningocele ofrece resultados satisfactorios, con beneficios significativos para los fetos y su vida futura, y que los riesgos para la madre y el feto son aceptables (13). La experiencia demuestra que las madres quedan muy conformes con los resultados obtenidos, aun cuando la recuperación posterior a la cirugía algunas veces haya sido más laboriosa (14). 
El feto como paciente: diferentes posturas sobre un mismo concepto

\section{El feto como paciente}

Uno de los retos bioéticos en perinatología es la utilización de la tecnología no invasiva y/o invasiva que proporcione información sobre la salud fetal; que dé orientación para su manejo terapéutico y genere bienestar materno-fetal (15). La dependencia materna que presenta el feto y la necesidad de pasar a través del cuerpo de la madre ante los procedimientos diagnósticos y tratamientos hace que, aunque infrecuentes, puedan existir conflictos potenciales que pongan en tela de juicio la condición del feto como un paciente (16). Conocer cómo los avances en el diagnóstico prenatal influyen directamente en el cuidado de la mujer embarazada, ubica al feto como paciente, al establecer comunicación con él para conocer su estado de salud. Con todo esto permitirá generar un compromiso médico ético y social, que tiende a evitar intervenciones agresivas sobre la madre y el feto con respeto a la dignidad humana de los mismos (15). Por los riesgos existentes, puede considerarse que no podría hablarse de una obligación médica de intervenir siempre; consideramos que esta postura también sería errónea y extrema (17).

El primer componente del enfoque integral propuesto para el análisis ético de la cirugía fetal es el concepto ético del feto como paciente. Este concepto fue empleado por Chervenak y McCullough, al plantear un marco ético para la medicina perinatal (18). Estos autores consideran que el feto puede ser considerado paciente, aunque no le atribuyen un estatus moral independiente. Sostienen que existe un enlace entre el feto y su futuro estatus moral.

Es decir, plantear que el feto es un paciente significaría reconocer que el feto es una persona. Las diversas posturas que se han dado a lo largo de la historia de la medicina demuestran que no todos respaldarían esta afirmación (19).

En 1948, la Declaración Universal de los Derechos Humanos estableció en su artículo $3^{\circ}$ : «Todo individuo tiene derecho a la vida, a la libertad y a la seguridad de su persona» (20). Éste es un dato claro en la defensa del derecho de toda persona a la vida. La

Medicina y Ética - Octubre-Diciembre 2021 - Vol. 32 - Núm. 4 
discusión actual gira en torno a cuándo un ser humano comienza a ser persona.

Se puede explicar el concepto del feto como paciente desde tres visiones o fundamentaciones filosóficas sobre el concepto de persona y de las obligaciones que se deben tener hacia ella (estatus moral). Para saber si un feto es un paciente o no, se debe establecer si el feto tiene un estado moral independiente. Esto implica que las características que posee el feto son independientes de la madre o de cualquier otro factor y que, por lo tanto, generan obligaciones hacia el feto por parte de la madre y de su médico (21). Analizaremos tres posturas que emplean diversas fundamentaciones para determinar si el feto es un paciente.

\section{a) Fundamentación ontológica}

La antropología personalista ontológica, que defiende la sustancialidad de la persona y la sustancialidad del alma espiritual (22), permite explicar por qué el hombre es persona desde el momento de la concepción, o por qué el hombre es persona aunque esté en situación de menor integridad física, moral o intelectual (15). Éste sería el caso de los embriones y de los fetos, con o sin malformaciones.

Los filósofos clásicos hacían derivar la dignidad de la persona humana, no solamente de la intelectualidad o racionalidad de la naturaleza del hombre, o de la conciencia de sí, sino previamente de su dimensión de subsistencia. El «ser» se afirma principalmente en la sustancia; una substancia es propiamente «un ser»; o sea, aquello que existe en sí mismo o por sí mismo, o aquello que subsiste por sí mismo y no en otro. Sólo el individuo subsistente poseedor de la naturaleza racional puede ser denominado persona (15) y, en cuanto persona, tiene derechos, independientemente de la funcionalidad o del ejercicio de la racionalidad que pueda poseer.

La fundamentación ontológica de la persona no reduce a la misma a sus actos específicos (presentes o futuros), sino que acepta la 
existencia de la persona, en tanto substancia, cuando sus actos aún no reflejan todas sus capacidades, ya sea por falta de desarrollo, como lo sería el embrión, o cuando sus capacidades ya desarrolladas no pueden expresarse, por una discapacidad física o intelectual que sobreviene accidentalmente (15). Por este motivo, considera que el feto es paciente desde el mismo momento en que comienza a existir, tras la fusión de los gametos masculino y femenino.

Moratalla (1) concuerda con esta fundamentación ontológica, y afirma que la medicina perinatal considera al feto como paciente, al que se puede no sólo diagnosticar mejor y con mayor precisión, sino también tratar. El feto como paciente está en fases de especial fragilidad y vulnerabilidad, necesitando por ello una atención muy especializada. La terapia prenatal busca un beneficio para el no nacido y que la intervención sea de bajo riesgo. Está comúnmente aceptado que, para ofrecer cualquier procedimiento a favor de la salud o bienestar prenatal, se deben asegurar la probabilidad de curación o los beneficios potenciales, la seguridad de la intervención, basada en modelos experimentales en animales, y la evaluación de los riesgos sobre la vida y la salud de la madre. Lógicamente se requiere el consentimiento de ella tras la información clara y objetiva sobre los riesgos y beneficios para ella y para el hijo (1). Estas condiciones están muy bien expresadas en los criterios IFMSS (criterios para la realización de cirugía fetal creados por los fundadores de la Sociedad Internacional de Medicina Fetal y Cirugía, en 1982).

La fundamentación ontológica se apoya en la información que nos ofrece la biología, que permite distinguir un nuevo ser a partir de la información genética del cigoto. A partir de ese momento hay un nuevo cuerpo (comprobable empíricamente), que posee su identidad, prosigue su propio ciclo vital (supuestas todas las condiciones necesarias y suficientes) bajo su control autónomo, que se autoconstruye en un proceso altamente coordinado, dictándose a sí mismo las direcciones de crecimiento, según el programa de su propio genoma (23). Este cigoto pasará, sin solución de continuidad, por las diversas etapas hasta llegar a un individuo adulto. Esa

Medicina y Ética - Octubre-Diciembre 2021 - Vol. 32 - Núm. 4 
identidad común, mantenida durante todo el desarrollo es la que lleva a afirmar que el feto es una persona (24). A partir de esta observación surge el reconocimiento de una dignidad intrínseca del feto, que lo hace merecedor de los derechos humanos (25) durante toda su existencia.

Moratalla describe el «diálogo molecular» entre el embrión recién concebido y su madre: Mientras recorre el camino hacia el útero, el embrión recién concebido libera moléculas de interlenquinas, que reciben los receptores especificos de las trompas de Falopio de la madre. Como repuesta, las trompas producen varias sustancias. Los llamados factores de crecimiento, que permiten el desarrollo embrionario. Los factores de supervivencia (inhibidores de la apoptosis o muerte celular programada), que inyectan la vitalidad que el embrión necesita porque, durante los 5 primeros días, no dispone de más energía que la guardada en el óvulo. El factor LIF, por tener receptores en las células del trofoblasto (la envoltura) del embrión, hace posible que sus células formen parte del sistema inmune en esta etapa de gestación; de forma que el trofoblasto empieza a actuar como la piel del embrión (26).

Como veremos a continuación, otras posturas niegan esa continuidad del ser humano, afirmando que sólo puede reconocerse la persona en determinados momentos de su existencia. Para estos autores, la persona se identificaría con las manifestaciones de capacidad intelectual, especialmente en la autorreflexión. Ejemplos de esta postura son los autores de orientación utilitarista (27).

\section{b) Fundamentación funcionalista}

A diferencia de la bioética personalista, se encuentran autores de corrientes funcionalistas como Peter Singer (28), que afirman que la ética se extiende a todos los seres dotados de sensibilidad: Todos los seres que son capaces de sentir dolor y placer son considerados sujetos morales. Tomando en cuenta lo anterior, Singer coloca al feto (no al embrión) en igual estatus moral que un animal. También afirma que es inmoral permitir que sufran aquellos seres cuya capacidad mental es menor a la nuestra. Si el feto siente dolor, puede ser dañado en un sentido 
moral. A su vez, afirma, que el embrión no tiene ningún interés y, como otros organismos insensibles, no puede ser dañado en ningún sentido moral (29).

La Dra. Báez-Reyes, del Instituto Nacional de Perinatología-Clínica de Especialidades de la Mujer (México) (30), tiene una postura más a favor del feto: lo define como una «persona en potencia»: la unión del esperma y el ovocito le dan el potencial a un feto para llegar a ser una persona con razón moralmente relevante, y el término potencial es usado para describir una posibilidad para el feto, que es una persona en potencia en ese camino, siempre y cuando su crecimiento no sea afectado. También asegura que el feto, o también llamado individuo potencial, es un paciente diferente de los niños ya nacidos y desarrollados. Por este motivo, afirma con razón que los fetos enfermos tienen el derecho moral de ser atendidos y tratados cuando hay cura, previo consentimiento de los padres, siendo responsabilidad de los servicios de salud ofrecerles los beneficios de los sistemas médicos, con la calidad y calidez que sean necesarias. Sin embargo, asegura con palabras propias del neo-lenguaje actual y con un concepto de dignidad bumana propia del que piensa que existe un salto ontológico que convierte un individuo potencial en persona desarrollada que, ante un feto con defectos o enfermedades incompatibles con la vida, este último tiene el derecho a ser tratado con todo respeto y de acuerdo con la decisión de los padres de no prolongar su agonía más allá del nacimiento, con toda la carga de la asistencia médica y el encarnizamiento terapéutico que sólo logrará alargar su deteriorado y penoso proceso terminal; también tiene el derecho a una muerte digna en forma prenatal (28).

Chervenak y McCullough (31) pueden ser incluidos en este grupo, aunque también podrían ser incluidos en el tercero (porque recurren a conceptos principialistas). Afirman que el estatus moral del feto depende de si se espera de manera confiable que más adelante logre el estatus moral relativamente inequívoco de convertirse en un niño y, aún más tarde, el estatus moral de convertirse en una persona (29). Se podría decir que es un reconocimiento «condicional». El feto es un paciente y, por lo tanto, una persona, cuando se prueba confiablemente que tiene oportunidad de convertirse en un 
niño (29). Ellos sostienen que poseer un estatus moral significa que otros tienen la obligación de proteger y promover los intereses de esa entidad. Están en desacuerdo con la afirmación de que un feto tiene un estatus moral independiente de otras entidades, incluidas la mujer embarazada, el médico y el estado, lo que generan obligaciones de los demás hacia él (29).

Estos autores explican que el primer vínculo entre un feto y su posterior estatus moral como niño y luego como persona, es la viabilidad extra-uterina (20). Cuando el feto es viable y la mujer embarazada se presenta al médico (que cuida formalmente de su embarazo), es allí cuando, según la visión de estos autores, el feto se convierte en un paciente (29). La segunda condición que hace que un feto salte ontológicamente para convertirse en un niño y luego en persona, es la decisión de la mujer embarazada de continuar un embarazo pre-viable hasta su viabilidad y, por lo tanto, a término (29). En consecuencia, los criterios éticos para guiar la innovación en la cirugía fetal deben tener en cuenta las obligaciones basadas en la beneficencia para el paciente fetal (adjudicado por su viabilidad y la decisión materna) y las obligaciones basadas en la beneficencia y la autonomía para la mujer embarazada (29). Briozzo et al. (16), citando a Chervenak y McCullough, afirman que es la mujer embarazada la que presenta al feto como paciente, aunque esto no lo convierte en sujeto de derechos automáticamente (16).

El Dr. Sebastiani (20), con una visión semejante a los dos autores anteriores, afirma que la «viabilidad» es el primer «sentido» ético que tiene el feto como paciente (20).

La viabilidad no es una propiedad intrínseca del feto, debido a que debe ser comprendida biológica y tecnológicamente. Es en virtud de estos dos factores que el feto puede ser viable y puede existir fuera del útero y convertirse en un niño. Ambos factores no son dependientes, ni existen en función de la autonomía de la madre. Por lo tanto, el feto es un paciente cuando alcanza una madurez suficiente para sobrevivir al periodo neonatal, sea por sus propios medios o por medios asistidos. Dado que la viabilidad depende del lugar en el que tenga que nacer 
este feto, el concepto de viabilidad difiere según los lugares. El consejo dirigido a la madre para el beneficio del feto debe tener en cuenta la severidad de las anomalías fetales, la "prematurez» extrema y las obligaciones de la madre. Cuanto más severa es la anomalía fetal o las opciones de morir o de quedar con un déficit neurológico permanente menos dirigido será el consejo orientado hacia el beneficio fetal (20).

De acuerdo con los citados autores Chervenak y McCullough, y según la crítica realizada por Carlos Alberto Gómez Fajardo (32), habría situaciones en las que se daría la paradoja de un embarazo gemelar con uno de los fetos enfermo y el otro sano, en que uno de ellos sería paciente y el otro no, justificándose el feticidio selectivo del enfermo como una acción de carácter «terapéutico», coherente con la voluntad e intereses de la madre (30).

Por lo que hemos visto, los autores con fundamentación funcionalista ocupan un amplio abanico que va de la negación del feto como paciente hasta un reconocimiento relativo y condicionado. Niegan que el feto tenga una dignidad intrínseca, y algunos de ellos hacen depender su estatus moral de su pronóstico vital y de la aceptación de su madre (33).

Para estos autores, lo determinante es lo que la persona puede «hacer», y no lo que «es». Nos parece significativa la postura de Mills (2013), que afirma que ciertos fetos tienen «personalidad en potencia» (que todavía no puede ser ejercitada) y, por lo tanto, no son personas (34). Desde nuestro punto de vista, la personalidad no es en potencia: es o no es. Lo que está en potencia, en ese caso, es la posibilidad de ejercer los atributos personales.

\section{c) Fundamentación principialista}

Los autores principialistas elaboran un «paradigma» moral para quienes trabajan en el ámbito de la salud, a fin de proporcionar una referencia práctica y conceptual que pueda servir de orientación en situaciones concretas (35). Tal paradigma está asentado en las bases de los principios de autonomía, beneficencia, no maleficencia y 
justicia, interpretados a la luz de dos teorías, el utilitarismo mitigado y la deontología prima facie (33).

Ejemplo de este tipo de perspectiva ética es la de Dickens y Cook (36), los cuales analizan las implicaciones éticas del concepto de feto como paciente desde dicha visión. Si bien están de acuerdo en la beneficencia y no maleficencia hacia el feto y la madre, hacen prevalecer el principio de autonomía materna como una forma de respetar el principio de justicia cuando existen conflictos de intereses. Ellos sostienen que los médicos han desarrollado el concepto de tratar a los fetos como si fueran pacientes, no con el objeto de subordinar a las pacientes embarazadas a los intereses del feto, sino para prevenirse de las significativas repercusiones que el tratamiento de la mujer embarazada pueda tener sobre el feto. Dickens y Cook aseguran que este concepto refleja varios principios éticos claves, incluyendo el principio ético bistórico de «no hacer daño» (no maleficencia); el deber positivo de hacer el bien permitiendo a las pacientes ejercitar su derecho a elegir de forma protectora y para el beneficio de los bijos que pretenden tener (beneficencia), y ambos como elementos centrales del principio referente al respeto a los pacientes (34). Esto permite a las mujeres embarazadas conservar su autonomía, ya que toman decisiones informadas, permitiendo, a su vez, la protección de los más vulnerables. Las mujeres que dependen del tratamiento y de la información médica son vulnerables, así como lo son los fetos en riesgo de daño, debido a decisiones médicas mal informadas, tomadas por los proveedores de salud y sus pacientes (34).

La visión de Dickens y Cook sobre la administración de justicia reconoce la dependencia del feto, pero considera a la paciente embarazada en igualdad, ya no con el feto, sino con aquellas pacientes que no están embarazadas (ejemplo: otros familiares). Esto constituye la base en la cual la ley británica permite expresamente la opción del aborto por los efectos que la continuación del embarazo pudiera traer sobre la salud de los hijos nacidos de la mujer embarazada (34).

Rodrigues Catarina et al. critican el marco ético para la medicina perinatal de Chervenak y McCullough (Chervenak y McCullough, 2003). Sostienen que, desde el punto de vista del principialismo, 
deben tomarse en cuenta dos principios: autonomía y beneficencia. Entienden que, por encima del principio de beneficencia a favor del feto, se encuentra el principio de autonomía de la madre. Esa autonomía permite a la embarazada otorgar y retirar la condición de paciente propia y del feto. Por este motivo, consideran que el estatus moral de la mujer es superior al del feto. La conclusión es que el feto no es paciente en el mismo grado de su madre (37). En cambio, De Vries sostiene la postura contraria: la beneficencia del feto debería estar por encima de la autonomía de la madre, evitando el supuesto conflicto entre la madre y su hijo, a través del concepto de respeto (38).

Radic et al. reconocen que hay un amplio debate acerca del estatus personal del feto. Afirman que, de acuerdo con la postura que se adopte sobre ese estatus personal, dependerá si se considera paciente sólo a la madre o también al feto. Incluso reconocen que algunos autores estiman que la madre sería una «voluntaria altruista» y el verdadero paciente sería el feto. Para estos autores, dado que tanto la madre como el feto están íntimamente relacionados, y ambos sufren la intervención, ambos deben ser considerados pacientes (39).

Puede apreciarse que el principialismo presenta la dificultad de la ausencia de una antropología que sirva de referencia. Esto lleva a que, según el punto de vista, se dé preferencia a un principio u otro, entendiéndolos como si uno compitiera con el otro (40). Además, tanto Beauchamp y Childress como sus seguidores, han derivado hacia una preponderancia de la autonomía del paciente, en detrimento del principio de beneficencia (41).

\section{Conclusión}

La fundamentación ética que considera que todo ser humano es persona y, por lo tanto, digna y merecedora de derechos desde su concepción hasta su muerte natural se basa en la continuidad del

Medicina y Ética - Octubre-Diciembre 2021 - Vol. 32 - Núm. 4 
ser, el cual no varía con las etapas del desarrollo ni con los accidentes que le acontecen al individuo. Esta posición considera que el feto es una persona con estatus moral independiente y, por lo tanto, se convierte en otro paciente diferente de su madre. Respeta la vida y la dignidad del feto.

Las corrientes bioéticas que conceptualizan a la persona y su dignidad según las funciones que ésta pueda cumplir, la viabilidad o el estado de desarrollo en el que se encuentren, comparten el concepto de feto como un paciente, pero su estatus moral dependerá de un tercero. En este caso, sólo es considerado paciente si la madre presenta al feto ante el médico para su cuidado. Esta postura prefiere el aborto de pacientes malformados como una acción compasiva hacia los padres y el propio feto.

El principialismo coincide con la idea de respetar la dignidad del feto como paciente, guiado por los principios de beneficencia y no maleficencia, pero prioriza la autonomía materna sobre los principios antes mencionados. Por este motivo, consiente acciones contra la vida del no nacido por una cuestión que considera de justicia hacia la madre, quien puede elegir el aborto eugenésico como solución. La diferencia que se observa respecto de la autonomía materna entre la visión personalista y la principialista es que, en el caso de la primera, la libertad materna no está por encima de la vida y dignidad del hijo, y que su autonomía se manifiesta por la elección de que se realice o no una cirugía a través de ella. Su negativa también es aceptable.

Las visiones éticas como la funcionalista o la principialista, que no consideran al feto como persona y, por lo tanto, como paciente con estatus moral independiente de la mujer embarazada, hacen posibles conductas que atenten contra la vida, promuevan conductas eugenésicas e intolerantes a la discapacidad. Estas posturas distan de la ontológica, en la cual el valor de una persona no es accidental ni cambiante, como tampoco sujeta a la opinión de terceros. 
El feto como paciente: diferentes posturas sobre un mismo concepto

\section{Referencias bibliográficas}

1. López Moratalla N. Avances de la medicina perinatal y la creciente intolerancia a la discapacidad. Cuadernos de Bioética. 2012; 23(2): 529-564. Disponible en: http://www.redalyc.org/articulo.oa?id=87524464010

2. Matar A. Hansson MG. Höglund AT. Values and value conflicts in implementation and use of preconception expanded carrier screening: An expert interview study. BMC Med Ethics. 2019; 20(1): 25. https://doi.org/10.1186/s12910-019-0362-1

3. Stapleton G, Dondorp W, Schröder-Bäck P, De Wert G. Just choice: A Danielsian analysis of the aims and scope of prenatal screening for fetal abnormalities. Med Health Care Philos. 2019; 22(4): 545-555.

https://doi.org/10.1007/s11019-019-09888-5

4. Shea M. The quality of life is not strained: Disability, human nature, well-being, and relationships. Kennedy Inst Ethics J. 2019; 29(4): 333-366.

https://doi.org/10.1353/ken.2019.0029

5. Best M. The dilemma of prenatal screening. Ethics \& Medicine. 2018; 34(2): 113.

6. Nuccetelli S. Abortion for fetal defects: Two current arguments. Med Health Care Philos. 2017; 20(3): 447-450. https://doi.org/10.1007/s11019-017-9765-2

7. Campbell SM, Stramondo JA. The Complicated relationship of disability and well-being. Kennedy Inst Ethics J. 2017; 27(2): 151-184.

https://doi.org/10.1353/ken.2017.0014

8. Mayo Clinic. Rochester. (Consultado el 28 de febrero de 2021). Disponible en: https://www.mayoclinic.org/es-es/tests-procedures/fetal-surgery/about/pac20384571.

9. Wenstrom KD, Carr SR. Cirugía fetal: principios, indicaciones y evidencia. Obstet Gynecol. 2014; 124: 817-835. https://doi.org/10.1097/AOG.0000000000000476

10. Knezevich M, Koehler SM, Wagner A. The Evolution of fetal surgery. Division of pediatric surgery, Department of Surgery, Medical College of Wisconsin; Milwaukee, WI. 2017. Disponible en: www.openaccesspub.org.

11. Huamán Guerrero M. Historia de la cirugía fetal. Rev Peru Ginecolog Obstet. 2019; 65(4): 479-485. https://doi.org/10.31403/rpgo.v65i2208

12. Vuletin F. Nuevos desafíos en cirugía fetal. Rev. Chil. Pediatr. 2013; 84(3): 254-261. http://dx.doi.org/10.4067/S0370-41062013000300002

13. Antiel RM, Collura CA, Flake AW, et al. Physician views regarding the benefits and burdens of prenatal surgery for myelomeningocele. J Perinatol. 2017; 37(9): 994-998. https://doi.org/10.1038/jp.2017.75

14. Fry JT. Frader JE. "We want to do everything»: How parents represent their experiences with maternal-fetal surgery online. J Perinatol. 2018; 38(3): 226-232. https://doi.org/10.1038/s41372-017-0040-4

15. Lukac de Stier, ML. La antropología tomista de Guillermo Blanco. Sapientia. 2011; 67: 229-230. Consultado en: http://bibliotecadigital.uca.edu.ar/repositorio/revistas/antropologia-tomista-blanco-stier.pdf

Medicina y Ética - Octubre-Diciembre 2021 - Vol. 32 - Núm. 4

1005

https://doi.org/10.36105/mye.2021v32n4.03 
16. Briozzo L, et al. Abordaje clínico del conflicto de interés materno fetal y su relación con el estatus del feto como paciente. Rev Méd Urug. 2013; 29(3): 187-194.

17. Kanaris $C$. Foetal surgery and using in utero therapies to reduce the degree of disability after birth. Could it be morally defensible or even morally required? Med Health Care Philos. 2017; 20(1): 131-146.

https://doi.org/10.1007/s11019-016-9727-0

18. Chervenak FA, McCullough LB. The fetus as a patient: An essential concept for the ethics of perinatal medicine. Am J Perinatol. 2003 Nov; 20(8): 399-404. https://doi.org/10.1055/s-2003-45383

19. Chervenak FA, McCullough LB. An ethically justified framework for clinical investigation to benefit pregnant and fetal patients. Am J Bioeth. 2011; 11(5): 39-49. https://doi.org/10.1080/15265161.2011.562595

20. ONU. Declaración Universal de los Derechos Humanos. Ginebra 1948. (Consultado el 19 de marzo de 2021). Disponible en: https://www.un.org/es/about-usuniversal-declaration-of-human-rights

21. Sebastiani M. Análisis ético bajo el concepto del feto como paciente en los casos de anencefalia. Comité de Bioética del Hospital Italiano de Buenos Aires. Sep. 2001.

22. García JJ. Bioética personalista y bioética principialista. Perspectivas. Cuadernos de Bioética. 2013; 24(1): 67-76.

23. Melina L. El embrión humano: estatuto biológico, antropológico y jurídico. Actas del Congreso Internacional de Bioética. Bioética y dignidad en una sociedad plural. Pamplona 1999. (Consultado el 19 de marzo de 2021). Disponible en: https://www.unav.edu/web/unidad-de-humanidades-y-etica-medica/material-debioetica/congreso-internacional-bioetica-1999/el-embrion-humano\#cap1

24. Arango Restrepo P. Estatuto del embrión humano. Escritos. 2016; 24(53): 307-318. https://doi.org/10.18566/escr.v24n53.a04

25. Sulmasy D.P. The varieties of human dignity: A logical and conceptual analysis. Med Health Care and Philos. 2013; 16: 937-944.

https://doi.org/10.1007/s11019-012-9400-1

26. López Moratalla N. Comunicación materno filial en el embarazo. Cuad. Bioét. XX. 2009; 3: 303-315.

27. Levin SB. Upgrading discussions of cognitive enhancement. Neuroethics. 2016; 9: 53-67. https://doi.org/10.1007/s12152-016-9253-z

28. Singer P. Ética práctica. Barcelona: Ariel, 1991.

29. Flores Muñoz MA. Las intervenciones en el feto, el dolor y sus dilemas bioéticos. Perinatol. Reprod. Hum. 2014; 28(2): 114-118. Disponible en: http:// www.scielo.org.mx/pdf/prh/v28n2/v28n2a8.pdf

30. Báez Reyes MR. El feto y los derechos humanos. Rev Sanid Milit Mex. 2010 May-Jun; 64(3): 125-132.

31. Chervenak F, McCullough L. Ethics of fetal surgery. Clin Perinatol. 2009; 36. 237-246. https://doi.org/10.1016/j.clp.2009.03.002 
El feto como paciente: diferentes posturas sobre un mismo concepto

32. Gómez Fajardo CA. Anotaciones críticas sobre algunos sofismas en medicina materno-fetal. Revista Lasallista de Investigación. 2014; 11(1), 152-160.

https://doi.org/10.22507/rli.v11n1a18

33. Selgelid M.J. Moral uncertainty and the moral status of early human life. J Med Ethics. 2013 May; 39(5): 324. https://doi.org/10.1136/medethics-2012-101164d

34. Mills E. Early abortion and personal ontology. Acta Anal. 2013; 28: 19-30. https://doi.org/10.1007/s12136-012-0182-0

35. García JJ. Bioética personalista y bioética principialista. Perspectivas. Cuadernos de Bioética XXIV. 2013/1 ${ }^{\text {a }}$.

36. Dickens BM, Cook RJ. Ethical and legal approaches to «The fetal patient». 83 International Journal of Gynecology and Obstetrics. 2003; 85-91.

https://doi.org/10.1016/s0020-7292(03)00320-5

37. Rodrigues HC, Van Den Berg PP, Düwell M. Dotting the l's and crossing the T's: Autonomy and/or beneficence? The «fetus as a patient» in maternal-fetal surgery. J Med Ethics. 2013; 39(4): 219-223.

https://doi.org/10.1136/medethics-2012-100781

38. De Vries R. Obstetric Ethics and the invisible mother. Narrat Inq Bioeth. 2017; 7(3): 215-220. https://doi.org/10.1353/nib.2017.0068

39. Radic JAE, Illes J, McDonald PJ. Fetal repair of open neural tube defects: Ethical, legal, and social issues. Camb Q Healthc Ethics. 2019; 28(3): 476-487. https://doi.org/10.1017/S0963180119000409

40. Cohen S. The logic of the interaction between beneficence and respect for autonomy. Med Health Care Philos. 2019; 22(2): 297-304.

https://doi.org/10.1007/s11019-018-9876-4

41. Saad TC. The history of autonomy in medicine from antiquity to principlism. Med Health Care Philos. 2018; 21(1): 125-137.

https://doi.org/10.1007/s11019-017-9781-2

Esta obra está bajo licencia internacional Creative Commons Reconocimiento-NoComercial-Compartirlgual 4.0.

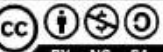

Medicina y Ética - Octubre-Diciembre 2021 - Vol. 32 - Núm. 4 
